\title{
New Tools for Easing System Integration, Deployment, Monitoring and Maintenance of Ocean Energy Devices
}

\author{
E. Omerdic, D. Toal and P. Finn \\ Electronic and Computer Engineering (ECE) Department \\ University of Limerick \\ Limerick, Ireland \\ Phone number: +353(0)61 202355, e-mail: edin.omerdic@ul.ie, daniel.toal@ul.ie, paddy.finn@ul.ie
}

\begin{abstract}
This paper describes a set of novel aiding tools and technologies for system integration, deployment, monitoring and maintenance of ocean energy devices. The main component of the system is smart remotely operated vehicle $\mathrm{ROV}_{\text {LATIS }}$ - a novel, multi-mode of operation marine robotics vehicle, developed at Mobile \& Marine Robotics Research Centre (MMRRC), UL for operational flexibility in highresolution near seabed survey from shallow inshore waters out to the continental shelf edge. The assistive visualisation and control tools, proposed in this paper, allow operators to have better situation awareness and enhanced vehicle control in presence of disturbances. Flexible design of these tools enables their use as separate modules, as well as their integration into unique system. Ocean energy technologies play an important part in meeting target that third of Irish electricity is generated from renewable sources by 2020. Proposed assistive tools could help developers of ocean energy devices in meeting this target during different stages of design, deployment and operation.
\end{abstract}

\section{Key words}

smart ROV, ocean energy, fault-tolerant control, realtime visualisation.

\section{Introduction}

Use of fossil fuels is continuously becoming more expensive. At the same time, their use as an energy source has a negative impact on the environment. On other side, Ireland has a vast range of high quality renewable energy resources. For example, theoretical and accessible levels of wave energy resources in Irish waters, estimated in [1], are about $525 \mathrm{TWh}$ and $21 \mathrm{TWh}$ respectively, while the overall electricity demand in Ireland for 2006 was estimated at 27.8 TWh. In addition, study [2] estimated viable levels of tidal energy resources in Ireland to be approximately $0.915 \mathrm{TWh}$ per year, considering only areas with tidal stream velocities of 2 $\mathrm{m} / \mathrm{s}$ or higher, due to current technology limitations.

As stated in [3], Ireland has set a target that renewable energy sources supply $33 \%$ of total electricity needs by 2020. The use of biomass, wind and ocean energy technologies is expected to play a dominant role in meeting this target.

The major obstacles restricting the development of wave and tidal energy devices include the following [4]:

- Technologies are in the developing phase, with little demonstration experience.

- Ocean energy devices must be able of withstanding extremely harsh weather conditions, which add to the cost of design and material.

- Deployment and maintenance costs may be relatively high because of locations where ocean devices are deployed.

- Unknown environmental issues associated with power generation from wave and tidal energy.

- Non-uniform grid connection and access (in most cases best locations for technology development usually have poor grid connection and access).

- The taxes on renewable energy technologies reduce the competitiveness of the technology.

This paper proposes a set of assistive tools and technologies that will address some of these issues and provide a framework for better planning, deployment, real-time monitoring and maintenance of ocean energy devices. The vision of the future for ocean energy devices [5] sees the complexity and costs of many of the systems deployed in the oceans increasing with time. With significant increase in costs and technical complexity it becomes of paramount importance to carry out as much engineering development, system testing, and scenario 'what-if' analysis in the design phase of these complex systems rather than in the roll out, deployment and operational phase. This will require the development of virtual ocean simulation environments and significant hardware-in-the-loop testing. For example, complete 
virtual reality simulation of the ocean energy devices, surface support vessels, ROVs, power connections, data transmission and physical characteristics can provide a virtual test bed prior to field deployment with associated cost benefits. Many integration problems could be detected, isolated and resolved in advance, yielding significant costs saving and improving the reliability of the overall system. In addition, the same test bed could be used to support enhanced real-time visualisations during deployment and maintenance operations.

At present, the typical displays presented to ROV operators during deployment and maintenance operations with ocean energy devices include low visibility realtime images from onboard cameras and optional 2D situation top view display(s). During such operations, the $\mathrm{ROV}$ is subject to disturbances, such as strong currents and waves. The assistive visualisation and control tools, proposed in this paper, provide operators with better situation awareness and enhanced vehicle control in the presence of disturbances, allowing them to concentrate on the task and to complete the work in a satisfactory manner and in a shorter time. The direct benefits of this approach are reduced maintenance and deployment costs due to savings in expensive support vessel time.

The outline of the paper is given in the following. An overview of the proposed system is given in section 2 . The system components are described in section 3 . Selected results of test trials are presented in section 4. Finally, section 5 summarizes the concluding remarks and provides directions for future work.

\section{System Overview}

The assistive tools and technologies are integrated into a system called the Virtual Underwater Lab for Ocean Systems Modelling and Simulation (Fig. 1). The System Core is built from a set of tools (Simulation Tools, Modelling Tools, Control Tools and Visualisation Tools), which provide easy and transparent interfaces between the Real-World Environment and the Virtual Environment. The Real-World Environment consists of the following real-world components:

- real ocean energy devices: wave energy point absorbers, tidal stream devices, etc.

- real ROVs: remotely operated vehicles, used for survey, deployment and maintenance of ocean energy devices.

- real support vessels: ships, boats etc. used as support platforms for launching/recovery of ROVs.

In the Real-World Environment each real-world component is subject to real-world disturbances (real waves and real ocean currents). Each real-world component has equivalent virtual component in the Virtual Environment. A virtual component is a mixed hardware/software real-time dynamic system, which is fully input-output compatible with corresponding realworld component on a signal level (Fig. 2). This means, for example, that the output message with navigation data for the virtual ROV is sent through the serial ports in the same format as the output of the real inertial navigation system (INS) installed on the ROV (identical message formats including checksums; identical RS232 port settings including baud rate, parity, number of stop bits, etc.). In this way the real-time controllers do not know the data source, i.e. if data is coming from the real-world or virtual instruments. This transparency of data sources, together with hardware-in-the-loop (HIL) control devices setup, provides a unique framework to design, develop and test the control system before the actual physical system components, such as ROV or ocean energy device, are built.

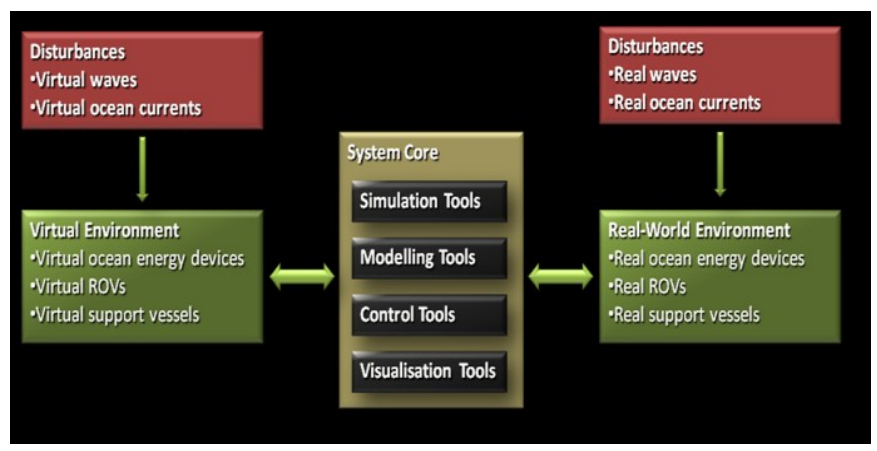

Fig. 1. Virtual Underwater Lab for Ocean Systems Modelling and Simulation

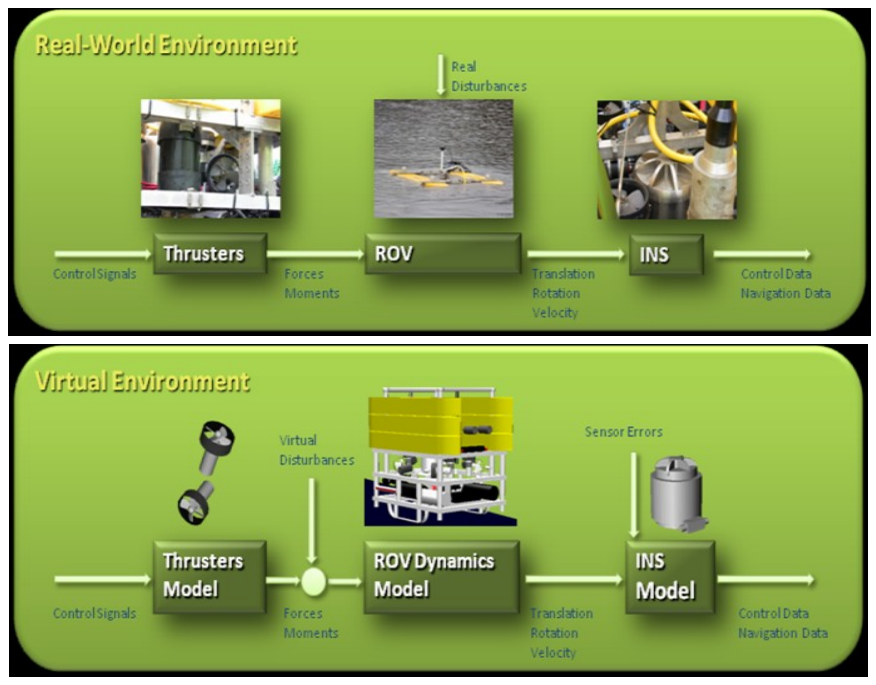

Fig. 2. Real ROV and Virtual ROV: input-output compatibility on a signal level

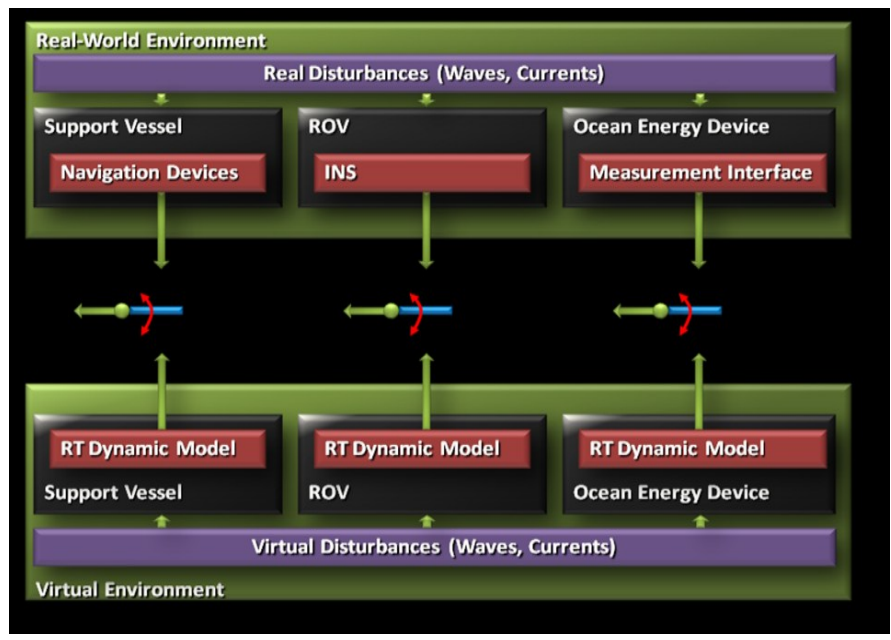

Fig. 3. Source selection for system data 
Software switches $\mathrm{S}_{1}, \mathrm{~S}_{2} \& \mathrm{~S}_{3}$ inside the System Core are used to select the source of navigation and measurement data for a support vessel, ROV and ocean energy device (Fig. 3). Depending on the position of switches $S_{1}, S_{2}$ \& $\mathrm{S}_{3}$, eight application modes can be distinguished, as illustrated in Fig. 4. Mode A was used during the $\mathrm{ROV}_{\text {LATIS }}$ development stage [6]. Mode $\mathrm{C}$ was used when the physical work on the ROV was completed, but the support vessel (ship) was not yet available. An interesting example of Mode E application is a pre-mission practice with the virtual ROV in a real-world environment (see section 4.A). Mode $\mathrm{G}$ was used during the real-world tests with the real ROV, real support vessel and virtual ocean energy device(s). Finally, Mode H could be used when real ocean energy devices become available.

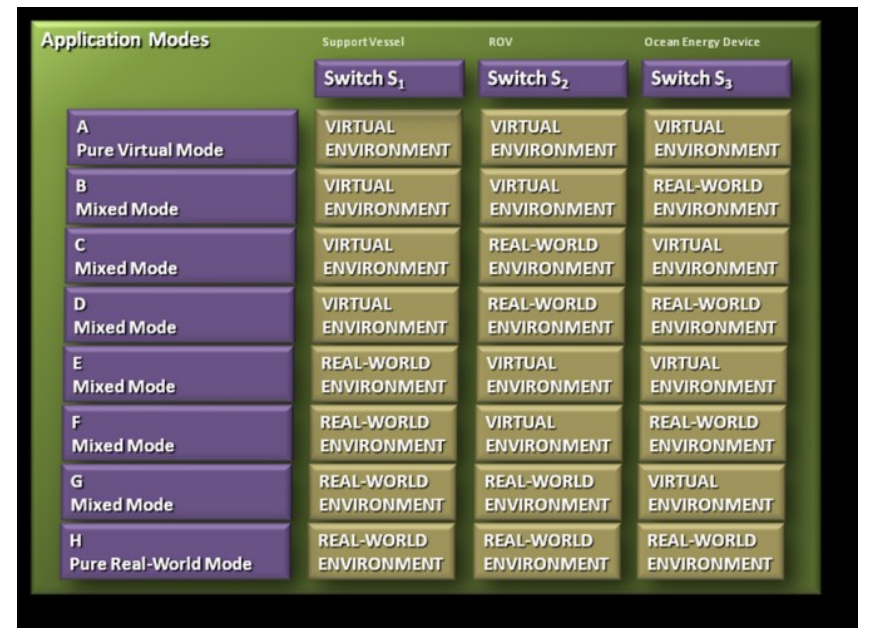

Fig. 4. Application Modes (possible settings of switches $\mathrm{S}_{1}-\mathrm{S}_{3}$ ).

In addition to input-output compatibility (electrical interface), there is also visual compatibility between realworld and corresponding virtual components, i.e. each real-world component is represented in virtual reality as a virtual model, with the same physical dimension and appearance (same colour etc.). Each virtual component is subject to virtual disturbances (virtual waves and virtual ocean currents). A full description of wave and ocean current models, used as virtual disturbances, is given in [7].

\section{System Components}

\section{A. Real-World Environment}

To date, the MMRRC team deployed the ROV $\mathrm{LATIS}_{\text {from }}$ the Celtic Explorer (Marine Institute) and Shannon One multi-cat (Shannon Port Company) research vessels. Network and serial interfaces have been developed for direct connections with navigation equipment on these support vessels. The existing library can be easily expanded to interface the System Core with other support vessels, like RV Celtic Voyager or similar.

Potential real-world ocean energy devices could be small-scale/full-scale prototypes, for example the Backward Bent Duct (Ocean Energy Ltd.), Wavebob (Wavebob Ltd.), McCabe Wave Pump (Hydam Ltd.) or a tidal stream turbine (OpenHydro).

\section{B. Virtual Environment}

Full virtual models of $\mathrm{ROV}_{\text {LATIS }}$, Celtic Explorer and the Shannon One multi-cat have already been developed and validated during test trials in Galway Bay (March 2009) and Limerick Dock (July 2009). Development of virtual components of ocean energy devices is work in progress. For demonstration purposes virtual generic tidal stream turbines have been developed. These virtual models are used in section 4.B to demonstrate possible system applications.

\section{System Core}

The System Core consists of hardware and software modules integrated into the Control Centre. The Control Centre can be located on a support vessel (for example, the Control Cabin - see Fig. 5) or onshore. Since the Ethernet network is used as the main communication medium, it is easy to expand the system by adding more monitoring points through the Internet.

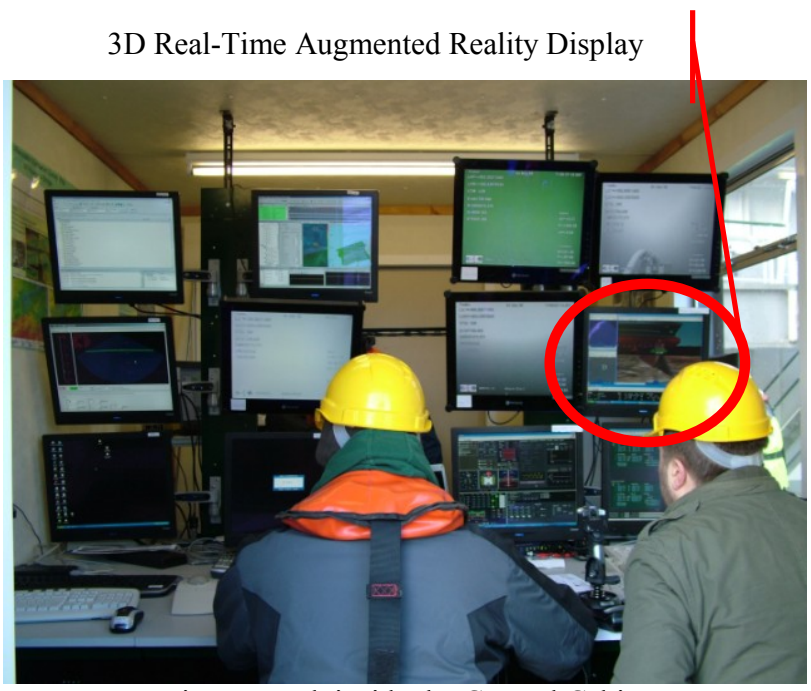

Fig. 5. Look inside the Control Cabin.

The development of the overall system has been implemented using a "V Diagram" product development cycle, based on rapid control prototyping (RPC) and hardware-in-the-loop (HIL) techniques, in order to make this cycle as efficient as possible. Software tools used for system development (LabVIEW, Matlab and Visual Studio $\mathrm{C}++$ ) offer a variety of advanced modelling, simulation, control design and visualisation tools in an interactive environment that helps the designer quickly evaluate a system without the need for prototype hardware. This capability provides the ability to determine specification, requirements, and modelling errors immediately instead of waiting for testing later in the design flow. Data outputs of individual components are bundled into clusters and transmitted using networkpublished shared variables, based on the NI PublishSubscribe Protocol (NI-PSP). The NI-PSP protocol uses less network bandwidth and is more efficient than TCP/IP for the given requirements of the NI-PSP protocol. Unlike the UDP protocol, however, the NI-PSP protocol guarantees delivery by implementing an additional layer of functionality on top of the raw UDP protocol. 


\section{ROV Fault-Tolerant Control System}

The $\mathrm{ROV}_{\text {LATIS }}$ control system utilises control allocation approach, where the actuator selection task (mapping of the total control efforts onto individual actuator settings) is separated from the regulation task (design of total control efforts) in the control design. A hybrid approach for control allocation [8] is based on integration of the pseudoinverse and the fixed-point iteration method. It is implemented as a two-step process. The pseudoinverse solution is found in the first step. Then the feasibility of the solution is examined analysing its individual components. If violation of actuator constraint(s) is detected, the fixed-point iteration method is activated in the second step. In this way, the hybrid approach is able to allocate the exact solution, optimal in the $l_{2}$ sense, inside the entire attainable command set. This solution minimises a control energy cost function, the most suitable criteria for underwater applications.

\section{System Validation}

The performance of the overall system has been successfully validated through a series of test trials in Galway Bay and Limerick Dock.

\section{A. Trials in Galway Bay}

Off shore trials (survey cruise; CE-09-04) of ROV were undertaken off the west of Ireland's Connemara coastline from 26 February to 6 March 2009. The vehicle was mobilised using the ship RV Celtic Explorer. The cruise consisted of a day in Galway port integrating and testing ROV and ship interfaces and six days at sea. During the trials all of the ROV's systems were proved, sensor interoperability was demonstrated and comprehensive vehicle diagnostics were performed. In addition, system identification was performed on the ROV and tuning of vehicle controllers was successfully carried out. Selected survey results are reported here.

\section{Enhanced 3D Real-Time Visualisation}

Fig. 6 displays $\mathrm{ROV}_{\text {LATIS }}$ in action during surface operations. Fig. 7 shows a screenshot captured at the same time from the 3D Real-Time Augmented Reality Display monitor inside the Control Cabin, showing the real-time measurements presented to the ROV pilot in the form of intuitive visual indicators, including 3D compass, desired and actual velocity vectors, trajectory trace etc.

\section{$\underline{\text { INS/DVL calibration }}$}

If a Doppler Velocity Log (DVL) is used as an aiding sensor for a Fibre Gyro Inertial Navigation System (INS), it has to be calibrated with respect to INS, prior to being used for navigation aiding. Speed inputs received from the DVL are measured in the DVL-reference frame without any pre-compensation. These inputs have to be compensated through an INS/DVL calibration procedure by calculating misalignment and scale factor between the INS and DVL reference frames.

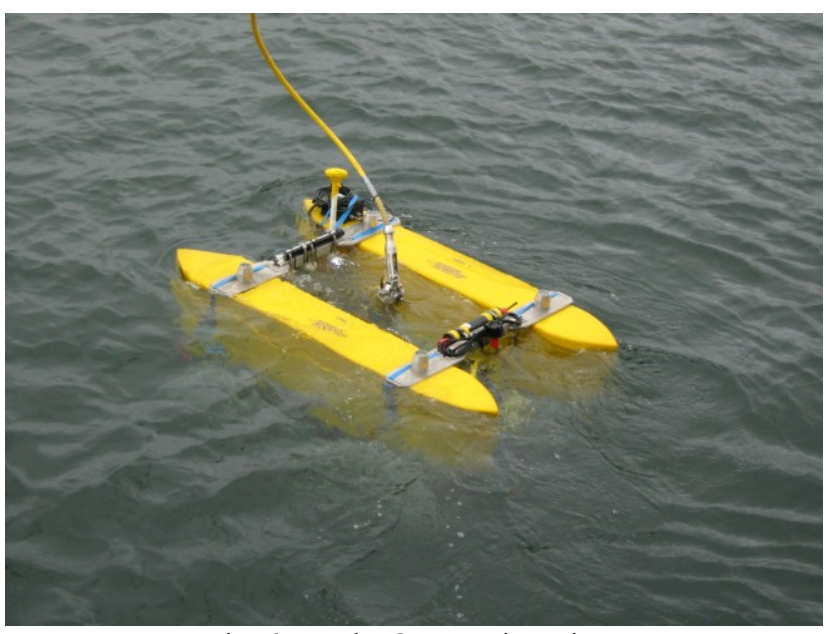

Fig. 6. Real ROV ${ }_{\text {LATIS }}$ in action

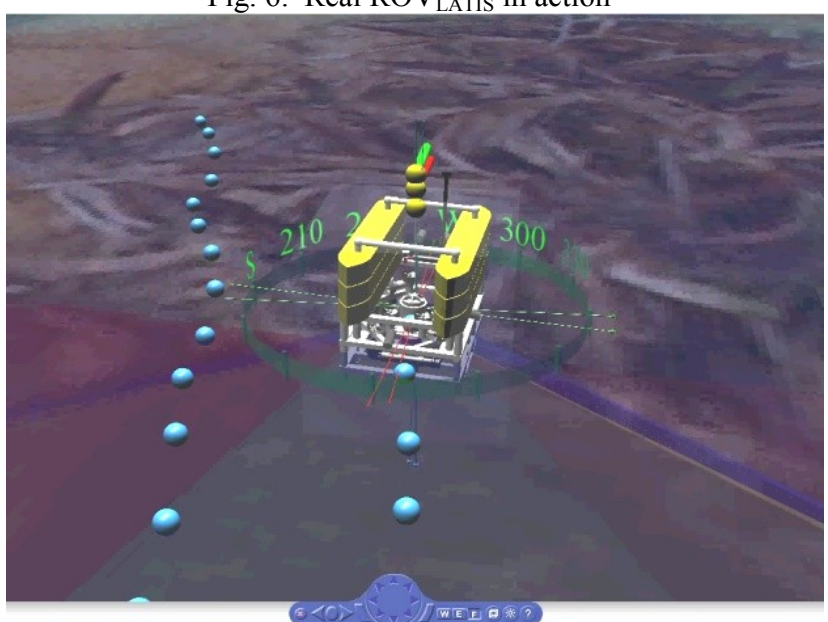

Fig. 7. 3D Real-Time Visualisation of ROV during survey
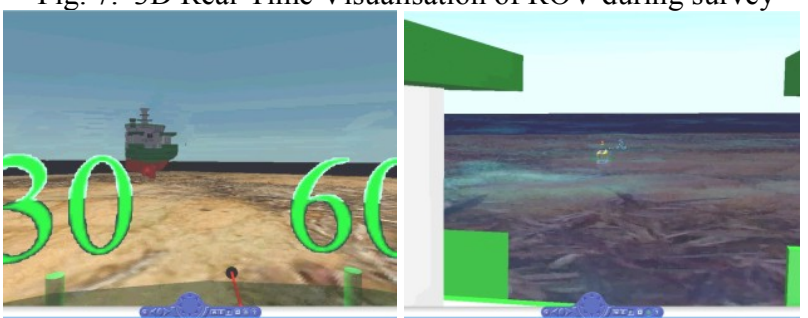

(a) Pilot Camera View

(b) View from ship's deck

Fig. 8. 3D Real-Time Augmented Reality Display: INS/DVL calibration with $\mathrm{ROV}_{\text {LATIS }}$ towed behind the ship

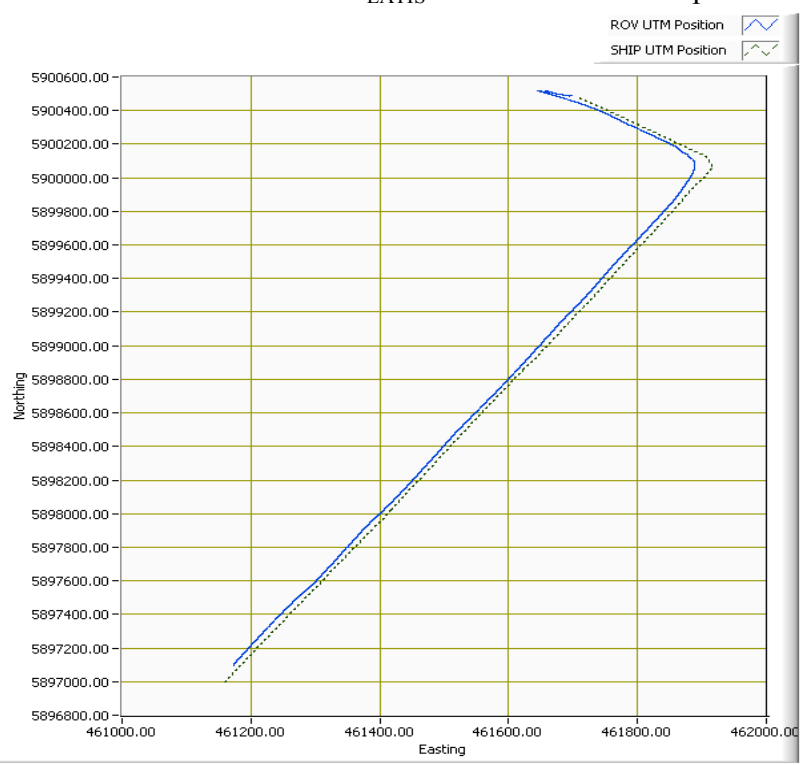

Fig. 9. ROV \& Ship trajectories during INS/DVL calibration 


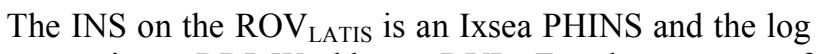
sensor is an RDI Workhorse DVL. For the purpose of this calibration a local DGPS receiver was mounted on the ROV, which was towed behind the ship in a straight line in shallow water on the surface for approximately 2 kilometres. ROV towing, as seen on the real-time Augmented Reality Display from different View Points, is shown in Fig. 8. ROV \& ship trajectories during INS/DVL calibration are shown in Fig. 9. After calibration the following values were obtained: Roll and Pitch misalignment: $0^{\circ}$ (both instruments are mounted on opposite sides of the same plate), Heading misalignment: $-44.654^{\circ}$ and Scale factor: $0.1 \%$.

\section{Pre-mission practice with virtual ROV and real ship}

The support vessel arrived on location in Galway Bay to perform vision experiments. Prior to deployment of the real ROV, Mixed Mode $\mathrm{E}$ was activated (switch $\mathrm{S}_{1}$ in position Real-World Environment and switches $\mathrm{S}_{2} \& \mathrm{~S}_{3}$ in position Virtual Environment, see Fig. 3 \& 4). In other words, the ship motion in the 3D Real-Time Augmented Reality Display was real, while ROV motion was simulated, i.e. while the real ROV was located on the deck, the virtual ROV was placed in the water and controlled by joystick. In this way the ROV pilot was able to practice the requirements imposed by mission objectives with the virtual ROV in a realistic environment, including diving to desired depth, following Figure of Eight trajectory etc. (see Fig. 10). The day after practising, switch $S_{1}$ was switched to position RealWorld Environment, the real ROV was deployed in the water and the vision experiments were realised without any trouble in a very satisfactory and smooth way.

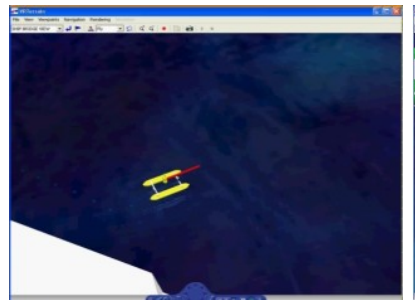

(a) View from deck

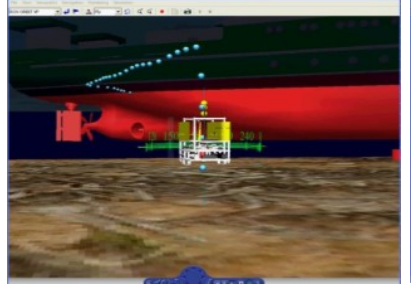

(c) View below surface mission practice with virtual ROV in realistic environment

\section{B. Trials in Limerick Dock}

Demo trials of ROV $\mathrm{LATIS}_{\text {were }}$ undertaken in Limerick Dock in the period from 1st - 3rd July 2009. The trials included an official launching ceremony, diving, wall \& gate video inspection, full multi-beam \& sidescan survey of the gate zone of the dock, testing of the thruster faulttolerant control system etc. Selected trial results are reported here.
Real pilot camera vs Virtual pilot camera

A comparison of real and virtual pilot camera views is shown in Fig. 12. The view from the real onboard pilot camera is shown in the top left monitor, while the corresponding view from the virtual pilot camera is shown in the top right monitor. The picture was taken between two missions, while the vehicle was on the deck. Due to independent open loop operator tilt control of real and virtual cameras, small difference in elevation can be observed, since the real camera was slightly tilted down, while the virtual camera was not tilted at all.

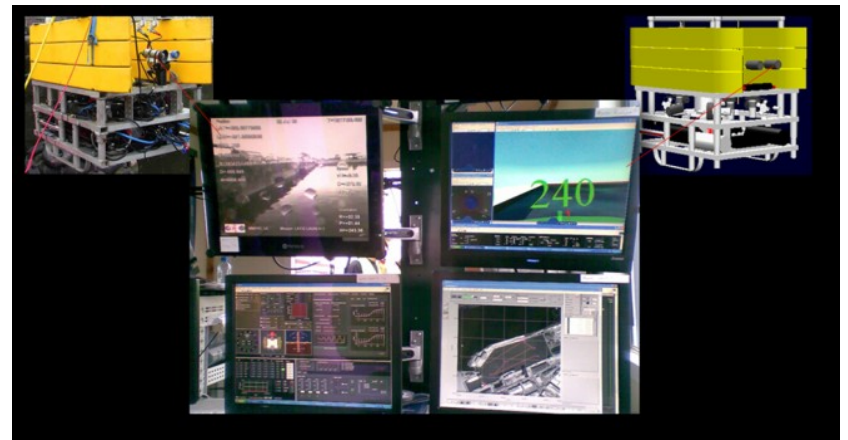

Fig. 12. Control Cabin: comparison of views from real and virtual pilot cameras

Evaluation of fault-tolerant features

In order to test the performance of a fault-tolerant control system, a number of test trials have been performed in Limerick Dock. The main task imposed on ROV control system was the path following with faulty thruster. The path was defined with a set of way-points. Different faulty situations were artificially generated by disabling thrusters in control software. Fig. 13 shows results from one of these tests. In this particular case, the front left horizontal thruster was disabled and the ROV was commanded to follow the rectangular path defined by way points WP1 - WP4 in surface mode of operation, with constant forward speed of $0.4 \mathrm{~m} / \mathrm{s}$. As indicated in Fig. 13, an excellent tracking performance has been achieved with only three working horizontal thrusters.

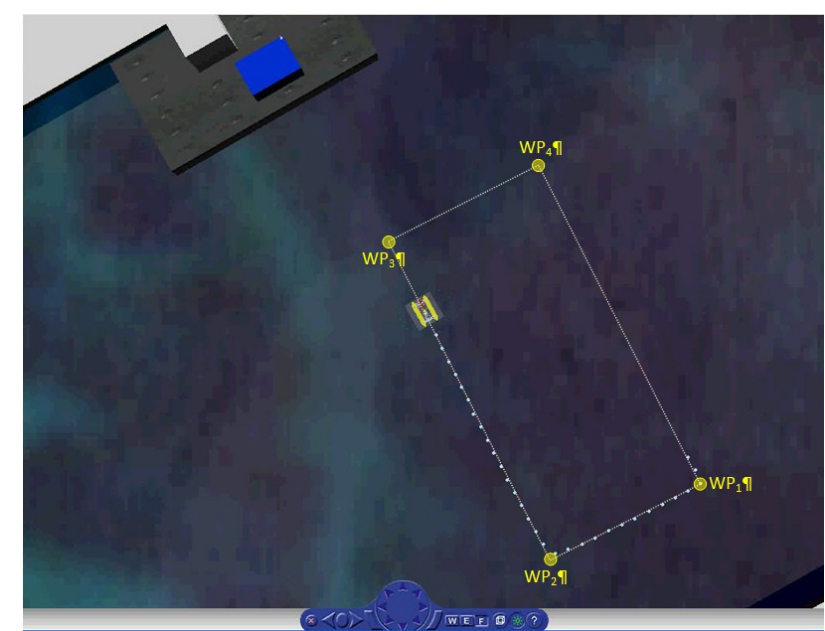

Fig. 13. 3D Real-Time Augmented Reality Display: Path following with faulty horizontal thruster

C. Real-time monitoring and maintenance of ocean energy devices (Virtual Environment)

In the following example virtual generic tidal stream devices were placed on the seabed and the typical 
maintenance mission has been simulated with a virtual ROV and a virtual support vessel (application mode is Mode A in Fig. 4). Simulated measurement and navigation data were processed with the System Core and used for real-time control and visualisation purposes. The mission objective was to simulate vehicle deployment, diving and inspection/maintenance under harsh conditions (strong waves and currents). Different sea states were simulated, with ITTC, JONSWAP and Torsethaugen spectrum types, different numbers of wave directions (short-crested and long-crested waves) etc.
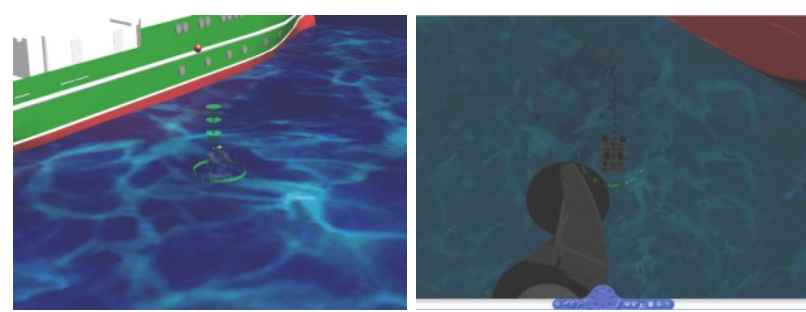

(a) DP in presence of waves

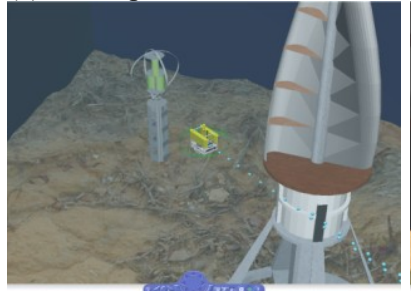

(b) View from seabed

(c) ROV and tidal devices

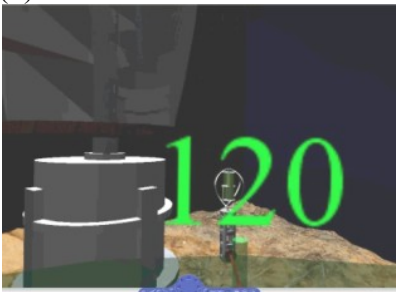
Fig. 14. 3D Real-Time Augmented Reality Display: Path following with faulty horizontal thruster

Ocean currents were simulated as layered time-varying 3D vector field, with different dominant directions in each layer. In all cases the control system was capable of performing given control tasks, such as keeping a given position and orientation, going to given position, etc. Different stages of a mission are indicated in Fig. 14, including dynamic positioning of the ROV on the surface in the presence of waves and currents, diving, dynamic positioning in close proximity to the ocean turbines, and viewing of the underwater scene from different view points with low and high visibility (a feature useful in training pilots).

\section{Conclusions \& Future Work}

This paper has presented a set of assistive tools and technologies for system integration, deployment, monitoring and maintenance of ocean energy devices. These tools are integrated into a system called the Virtual Underwater Lab for Ocean Systems Modelling and Simulation. The main components of the system are: the System Core; the Real-World Environment; and the Simulation Environment. Input-output compatibility between the real-world and virtual components enables easy switching between environments, creating opportunity for a variety of applications. Many integration problems can be detected, isolated and resolved in advance. Different mission scenarios can be designed and verified through the mixed use of virtual and real-world components, yielding significant costs savings and improving the reliability of the overall system.

Development of the Virtual Underwater Lab for Ocean Systems Modelling and Simulation is work in progress. Future work will include full development of virtual models of ocean energy devices, including real-time simulators of mechanical and electrical subsystems, development of interconnection interfaces compatible with the real-world devices etc. However, since ocean energy technology development is still in early and precommercial stages, it is expected there may be some difficulty in obtaining full descriptions of device interfaces for reasons of intellectual property protection and such as trade secret - knowhow, etc.

\section{Acknowledgements}

The development of ROV $\mathrm{VATIS}_{\text {and }}$ an the modelling and operations support tools described has been supported by funding under the Irish Marine Institute and the Marine RTDI Measure, Productive Sector Operational Programme, National Development Plan 2000 - 2006 (PhD -05-004, INF-06-013 and IND-05-03); Science Foundation Ireland under Grant Number 06/CP/E007 (Charles Parsons Energy Research Awards 2006); HEA PRTLI 3 (MSR3.2 project -Deep Ocean Habitat Mapping using and ROV; HEA PRTLI 4 Environment \& Climate Change Impacts and Responses Project/ Environment Graduate Programme; and Enterprise Ireland Commercialisation Fund Technology Development 2007 projects - MPPT Ring (CFTD/07/IT/313, "Multi-Purpose Platform Technologies for Subsea Operations) and PULSE RT (CFTD/07/323, "Precision Underwater Accelerated Sonar Emulation in Real Time).

\section{References}

[1] Sustainable Energy Ireland. Ireland's Wave Energy Resource, 2007.

[2] Sustainable Energy Ireland. Ocean Energy in Ireland. Ocean Strategy for Ireland submitted to the Department of Communications, 2005.

[3] Department of Communications, Marine and Natural Resources, Irish Government. Delivering a Sustainable Energy Future for Ireland, 2007. The Energy Policy Framework 2007 - 2020. Energy White Paper.

[4] Rourke, F.O., Boyle, F., and Reynolds, A. Renewable Energy Resources and Technologies Applicable to Ireland. Renewable and Sustainable Energy Reviews 13, 2009, pp. 1975-1984.

[5] Omerdic, E., Toal, D., Wallace, J., Ibrahimovic, I., Funnel, C., and Grehan, A. Marine Monitoring Platforms: Paradigms for Development in Ireland. Paperback, ISBN10: 1443813567, 2009 (Cambridge Scholars Publishing).

[6] Omerdic, E. and Toal, D. Smart ROVLATIS: From Design Concepts to Test Trials. In IFAC 8th Conference on Manoeuvring and Control of Marine Craft (MCMC'2009) Guarujá, Brazil, 2009.

[7] Omerdic, E. and Toal, D. Modelling of waves and ocean currents for real-time simulation of ocean dynamics. IEEE Oceans 2007, Aberdeen, 18-21 June 2007.

[8] Omerdic, E., Roberts, G., and Toal, D. Extension of Feasible Region of Control Allocation for Open-Frame Underwater Vehicles. CAMS 2004, Ancona. 